\title{
Hyperinsulinism and polycystic ovary syndrome (PCOS): role of insulin clearance
}

\author{
M. C. Amato ${ }^{1} \cdot$ R. $\operatorname{Vesco}^{1} \cdot$ E. Vigneri ${ }^{1} \cdot$ A. Ciresi $^{1} \cdot$ C. Giordano $^{1}$
}

Received: 5 May 2015 / Accepted: 24 July 2015 / Published online: 21 August 2015

(C) Italian Society of Endocrinology (SIE) 2015

\begin{abstract}
Purpose Insulin resistance and compensatory hyperinsulinism are the predominant metabolic defects in polycystic ovary syndrome (PCOS). However, hyperinsulinism, as well as being compensatory, can also express a condition of reduced insulin clearance. Our aim was to evaluate the differences in insulin action and metabolism between women with PCOS (with normal glucose tolerance) and age- and BMI-matched women with prediabetes (without hyperandrogenism and ovulatory disorders).

Methods 22 women with PCOS and 21 age/BMI-matched women with prediabetes were subjected to a Hyperinsulinemic-euglycemic clamp and an Oral Glucose tolerance Test (OGTT). Insulin sensitivity was assessed by the glucose infusion rate during clamp ( $M$ value); insulin secretion by Insulinogenic index, Oral Disposition Index (DIo) and $\mathrm{AUC}_{2 \mathrm{~h} \text {-insulin }}$ during OGTT; and insulin clearance by the metabolic clearance rate of insulin (MCRI) during clamp.

Results Women with PCOS showed significantly higher levels of $\mathrm{AUC}_{2 \mathrm{~h} \text {-insulin }}(p<0.011)$, Insulinogenic Index $(p<0.001)$, DIo $(p=0.002)$ and significantly lower levels of $\mathrm{AUC}_{2 \mathrm{~h} \text {-glucose }}(p=0.001)$. No difference was found between the two groups regarding insulin sensitivity ( $M$ value). Lower levels of MCRI were found in women with PCOS [420 (IQR 227-588) vs. 743 (IQR 597-888) $\left.\mathrm{ml} \mathrm{m}^{-2} \min ^{-1}: p<0.001\right]$. Furthermore, in the PCOS group, a strong independent inverse correlation was
\end{abstract}

C. Giordano

carlagiordano53@gmail.com; carla.giordano@unipa.it

1 Section of Endocrinology, Diabetology and Metabolism, Dipartimento Biomedico di Medicina Interna e Specialistica (DIBIMIS), University of Palermo, Piazza delle Cliniche 2, 90127 Palermo, Italy only observed between MCRI and $\mathrm{AUC}_{2 \mathrm{~h} \text {-insulin }}$ (PCOS: $\beta:-0.878 ; p<0.001$; Prediabetes: $\beta:-0.501 ; p=0.019)$. Conclusions Our study suggests that in normoglycemic women with PCOS there is peripheral insulin sensitivity similar to that of women with prediabetes. What sets PCOS apart is the hyperinsulinism, today still simplistically defined "compensatory"; actually this is mainly related to decreased insulin clearance whose specific causes and dynamics have yet to be clarified.

Keywords PCOS · Insulin clearance · Clamp · Hyperinsulinism · Insulin resistance

\section{Introduction}

Polycystic ovary syndrome (PCOS) represents an independent risk for the development of glucose intolerance states [1] and insulin resistance and compensatory hyperinsulinemia appear to be a central etiological characteristic in most women with PCOS [2-4], although it is not yet clear which of the two aspects plays a prominent role in the genesis of hyperandrogenism and ovulatory disorder. Furthermore, insulin resistance and hyperinsulinemia may represent two distinct features of the insulin disorder in PCOS [5]. In women with PCOS, as in the general population, the onset of impaired glucose tolerance or type 2 diabetes marks a failure of the pancreas to maintain this state of compensatory hyperinsulinemia $[6,7]$. For this reason, in PCOS insulin secretion should always be examined in the context of peripheral insulin sensitivity rather than in isolation [8]. In the general population, the relationship between insulin secretion and sensitivity is a constant hyperbolic function $[8,9]$ that can be quantitated by the Disposition Index (DI) [9]. DI is highly heritable [10], associated with 
specific genetic loci $[11,12]$ and the most powerful predictor of diabetes risk [13]. Unfortunately, there is no "gold standard" method for the evaluation of this aspect, although DI with insulin secretion assessed by the acute insulin response to glucose (AIRg) after an iv glucose bolus and insulin sensitivity assessed by Hyperinsulinemic-euglycemic clamp [14] or frequently sampled iv glucose tolerance test [FSIGT] [13] have been shown to predict the future development of T2D. Therefore, detailed study of these aspects can only be made using sophisticated and/or expensive diagnostic methods.

Another important issue that should be considered is the meaning of insulin serum levels, both fasting and after glucose stimulation. The measurements of peripheral levels of insulin do not always reflect the prehepatic concentration of hormones and do not elucidate potential actions of the liver on glucose metabolism; in addition, there is also a known large inter- and intra-individual variability in hepatic insulin extraction [15-18]. Therefore, in insulinresistant patients (such as women with PCOS), it is difficult to quantify not only "compensatory" pancreatic ability, but also the impact of hepatic, renal and tissue insulin clearance on peripheral serum insulin levels.

In this context, we evaluated the existence in women with PCOS of intrinsic decreased insulin clearance and/ or intrinsic compensatory pancreatic capacity, at the base of the condition of hyperinsulinism that characterizes the syndrome. For this purpose, we evaluated the differences in insulin action and metabolism between women with PCOS (with normal glucose tolerance) and age- and BMImatched women with prediabetes (without hyperandrogenism and ovulatory disorders).

\section{Materials and methods}

Fifty premenopausal Caucasian women [25 with PCOS according to the Rotterdam criteria [19] (but with normal glucose tolerance) and 25 with prediabetes matched for age and BMI (with Impaired Glucose Tolerance and/or Impaired fasting Glucose, without hyperandrogenism and ovulatory disorders)] followed up in our Day Hospital of Endocrinology (2010-2014) were recruited and subjected to both a Hyperinsulinemic-euglycemic clamp and an Glucose tolerance Test (OGTT). Three women with PCOS were excluded from the study as well as four women with prediabetes, who at the OGTT showed overt Diabetes (glycaemia to $120 \mathrm{~min}$ of the test $>11.1 \mathrm{mmol} / \mathrm{L}$ ). Twenty-two women with PCOS and 21 women with prediabetes were selected for the study. At screening (1 month prior to testing), standard diet and lifestyle advice were delivered and any treatment with metformin, insulin sensitizers, antiandrogens and hormonal contraceptives was suspended.
The following relevant data were obtained: family history of diabetes, oligo-amenorrhea, hirsutism, acne and age of menarche; weight, BMI, WC, blood pressure and Ferriman-Gallwey (FG) score (11 domains). Hirsutism was defined as FG score $>8$ [20]. In women with suspected PCOS (irregular menses and/or hirsutism), hyperandrogenemia was diagnosed by serum total testosterone, Sex Hormone Binding Globulin (SHBG) during the follicular phase (Day 7 from the beginning of the last period) and Free Androgen Index (FAI) was calculated as the ratio of total testosterone levels in nmol/l to SHBG levels in $\mathrm{nmol} / \mathrm{l} \times 100(\%)$ [21]. Biochemical hyperandrogenism was diagnosed when total testosterone $>2.84 \mathrm{nmol} / \mathrm{l}$, calculated on the basis of the 95th percentile upper limits of basal serum androgen normality in 144 healthy Sicilian eumenorrheal women without hirsutism and family history of PCOS (used as a control group in our previous study [22]). In women with PCOS transvaginal ovarian ultrasound scanning was performed between Days 5 and 10 after the beginning of the last period using a $7.5-\mathrm{MHz}$ vaginal probe transducer (General Electric LOGIQ 400MD, Milwaukee, WI, USA). Both ovaries were measured in the sagittal, transverse and coronal planes. Ovaries were classified as polycystic if 12 or more follicles measuring $2-8 \mathrm{~mm}$ in diameter were present in each ovary, and/or there was an increase in ovarian volume (>10 ml) [23].

On the same day, we also tested for total cholesterol, HDL cholesterol, LDL cholesterol and Triglycerides. Metabolic Syndrome (MetS) was diagnosed according to the National Cholesterol Education Program Adult Treatment Panel (NCEP-ATP) III definition [24] and diagnosis of diabetes mellitus according to the recommendations of the American Diabetes Association [25].

An OGTT was performed after a 12-h fast and blood samples were collected basally and after ingestion of $75 \mathrm{~g}$ glucose in $150 \mathrm{ml}$ water within 30, 60, 90 and $120 \mathrm{~min}$.

The Hyperinsulinemic-euglycemic clamp was performed under standard conditions [26], i.e. infusion of an insulin primer (Actrapid; Novo Nordisk, Bagsvaerd, Denmark) of $160 \mathrm{mU} / \mathrm{m}^{2}$ body surface for the first $4 \mathrm{~min}$ of the test, followed by $40 \mathrm{mU} / \mathrm{m}^{2}$ for the remaining $116 \mathrm{~min}$; venous fasting blood samples were collected, analyzed and stored as appropriate after arterialization. The rate of peripheral glucose utilization ( $M$ value) $\left(\mathrm{mg} \mathrm{Kg}^{-1} \mathrm{~min}^{-1}\right)$ was calculated by dividing the glucose amount infused during the last 40 min by body weight measured in kilograms (milligrams per kilogram per minute). Under the steadystate conditions of euglycemia, the glucose infusion rate equals glucose uptake by all the tissues in the body and is therefore a measure of tissue sensitivity to exogenous insulin. The metabolic clearance rate of insulin (MCRI) $\left(\mathrm{ml} \mathrm{m} \mathrm{m}^{-2} \mathrm{~min}^{-1}\right.$ ) was calculated as the insulin infusion 
rate divided by the steady-state plasma insulin level of the clamp [26].

$\beta$-cell function was determined by fasting insulin, Insulinogenic Index (the ratio of the incremental insulin concentration to the incremental glucose concentration at the 30 min sample) [27] and the $\mathrm{AUC}_{2 \mathrm{~h} \text { Insulin }}$ during OGTT. A composite indirect measure of $\beta$-cell function relative to insulin sensitivity, assessed by oral disposition index (DIo) [28], was calculated as ( $\Delta$ Insulin0$30 / \Delta$ Glucose $0-30) \times(1 /$ fasting insulin $)$. The trapezoidal method was used for the calculation of the areas under the curves for insulin $\left(\mathrm{AUC}_{2 \mathrm{hInsulin}}\right)$, and glucose $\left(\mathrm{AUC}_{2 \mathrm{hGlucose}}\right)$.

In all patients, we also calculated the visceral adiposity index (VAI), a sex-specific mathematical index based on anthropometric and metabolic parameters. VAI has been shown to be a useful tool for early detection of a condition of cardiometabolic risk before it develops into an overt metabolic syndrome [29], independently correlated with insulin sensitivity in various endocrine diseases characterized by a metabolic risk, in addition to PCOS [22], such as acromegaly [30] and prolactinoma [31]. The VAI was calculated as described [32] using the following sex-specific equations, where TG is triglyceride levels expressed in $\mathrm{mmol} / \mathrm{l}$ and HDL is HDL-Cholesterol levels expressed in $\mathrm{mmol} / \mathrm{l}$ :

Females : VAI $=\left(\frac{\mathrm{WC}}{36.58+(1.89 \times \mathrm{BMI})}\right) \times\left(\frac{\mathrm{TG}}{0.81}\right) \times\left(\frac{1.52}{\mathrm{HDL}}\right)$

This study was approved by the Institutional Review Board at the Faculty of Medicine of the University of Palermo and the identity of the participants remained anonymous during data analysis. At the time of the first observation in our Day Hospital of Endocrinology, all women regularly signed an informed consent for the scientific use of their data.

\section{Assays}

All hormones were measured in our laboratory using commercial kits. These included ELISA (DRG Diagnostics, DRG Instruments $\mathrm{GmbH}$, Germany) for total testosterone (ng/ml; analytical sensitivity: $0.083 \mathrm{ng} / \mathrm{ml}$; the intra- and interassay CVs were 3.28 and $4.73 \%$, respectively), and insulin (mUI/l; the intra- and interassay $\mathrm{CVs}$ were $\leq 4$ and $\leq 3.6 \%$, respectively). Chemiluminescence assays were used for serum SHBG (nmol/l; Immulite, Diagnostic Products, Genoa, Italy; analytical sensitivity: $0.015 \mathrm{nmol} / \mathrm{l}$; the intra- and interassay CVs were 5.50 and $6.20 \%$, respectively). Blood glucose levels $(\mathrm{mg} / \mathrm{dl})$ were measured using an electrochemical system (Glucocard, Menarini Diagnostics, Italy). Total cholesterol, HDL and triglycerides were measured in our laboratory using standard assays.
LDL cholesterol levels were calculated with Friedewald's formula. The conversion factors for the International System (SI) were the following: glucose $(\mathrm{mg} / \mathrm{dl} \mathrm{vs} . \mathrm{mmol} / \mathrm{l}$ : 0.0555), insulin (mUI/l vs. pmol/1: 6.945), total cholesterol (mg/dl vs. mmol/l: 0.0259), and total testosterone ( $\mathrm{ng} / \mathrm{ml}$ vs. nmol/l: 3.467$)$.

\section{Statistical methods}

The Statistical Packages for Social Sciences SPSS version 17 were used for the explorative data analysis. Normality of distribution for quantitative variables was assessed by the Shapiro-Wilk test. The quantitative variables not having a normal distribution were presented as median and interquartile Range (IQR); rates and proportions were calculated for categorical data. Differences between women with PCOS and women with Prediabetes in univariate analysis were detected by the Mann-Whitney $U$ test for the continuous variables and by the $\chi^{2}$-test and Fisher's exact test (when appropriate) for categorical variables. Two linear regression models were performed to investigate the variables that independently correlate with $\mathrm{AUC} 2_{\text {hInsu- }}$ lin. These variables were natural logarithmic transformed before being included in the regression models.

A $p$ value of $<0.05$ was considered statistically significant.

\section{Results}

The prevalence of women with insulin resistance (according to the recently proposed $M$ value cutoff of $4.9 \mathrm{mg} \mathrm{Kg}^{-1} \mathrm{~min}^{-1}$ ) [30] in the two groups was comparable: $19 / 21(90.5 \%)$ for women with prediabetes and $22 / 22(100 \%)$ for women with PCOS ( $p=0.223)$; indeed, women with PCOS showed a $M$ value comparable to the women with prediabetes [median (IQR); $1.28(0.85-1.62)$ vs. $\left.1.42(0.63-3.18) \mathrm{mg} \mathrm{Kg}^{-1} \min ^{-1} ; p=0.789\right]$. Also, no significant differences were found between the two groups for all anthropometric parameters studied (BMI, WC, Body Surface Area and VAI) (Table 1).

Regarding the metabolic profile women with PCOS had significantly lower levels of fasting glucose [4.72 (4.38$5.18)$ vs. $6.16(5.83-6.38) \mathrm{mmol} / \mathrm{l} ; p<0.001], \mathrm{AUC}_{2 \mathrm{hGlucose}}$ [700 (659-893) vs. $913(813-1029) \mathrm{mmol} \mathrm{l}^{-1} 120 \mathrm{~min}$; $p=0.001]$, MCRI [420 (227-588) vs. 743 (597-888) mmol $\left.1^{-1} 120 \mathrm{~min} ; p=0.001\right]$ and significantly higher levels of fasting insulin [132 (112.2-234) vs. 72.60 (54-108) $\mathrm{pmol} / 1 ; p<0.001$ ], $\mathrm{AUC}_{2 \mathrm{hInsulin}}$ [54594 (36828-118188) vs. 26730 (21060-35142) pmol 1-1 $120 \mathrm{~min} ; p<0.001]$, Insulinogenic index [218 (93.93-529) vs. 40.15 (19.42-71.42) $\mathrm{pmol} / \mathrm{mmol} ; p<0.001]$ and DIo [1.59 $(0.70-2.40)$ vs. 0.53 (0.28-0.94) $\left.\mathrm{mmol}^{-1} ; p=0.002\right]$ (Fig. 1). 
Table 1 Clinical and biochemical characteristics of women with PCOS (without glucose tolerance alterations) and women with prediabetes (women with impaired fasting glucose and/or impaired glucose tolerance and without PCOS)

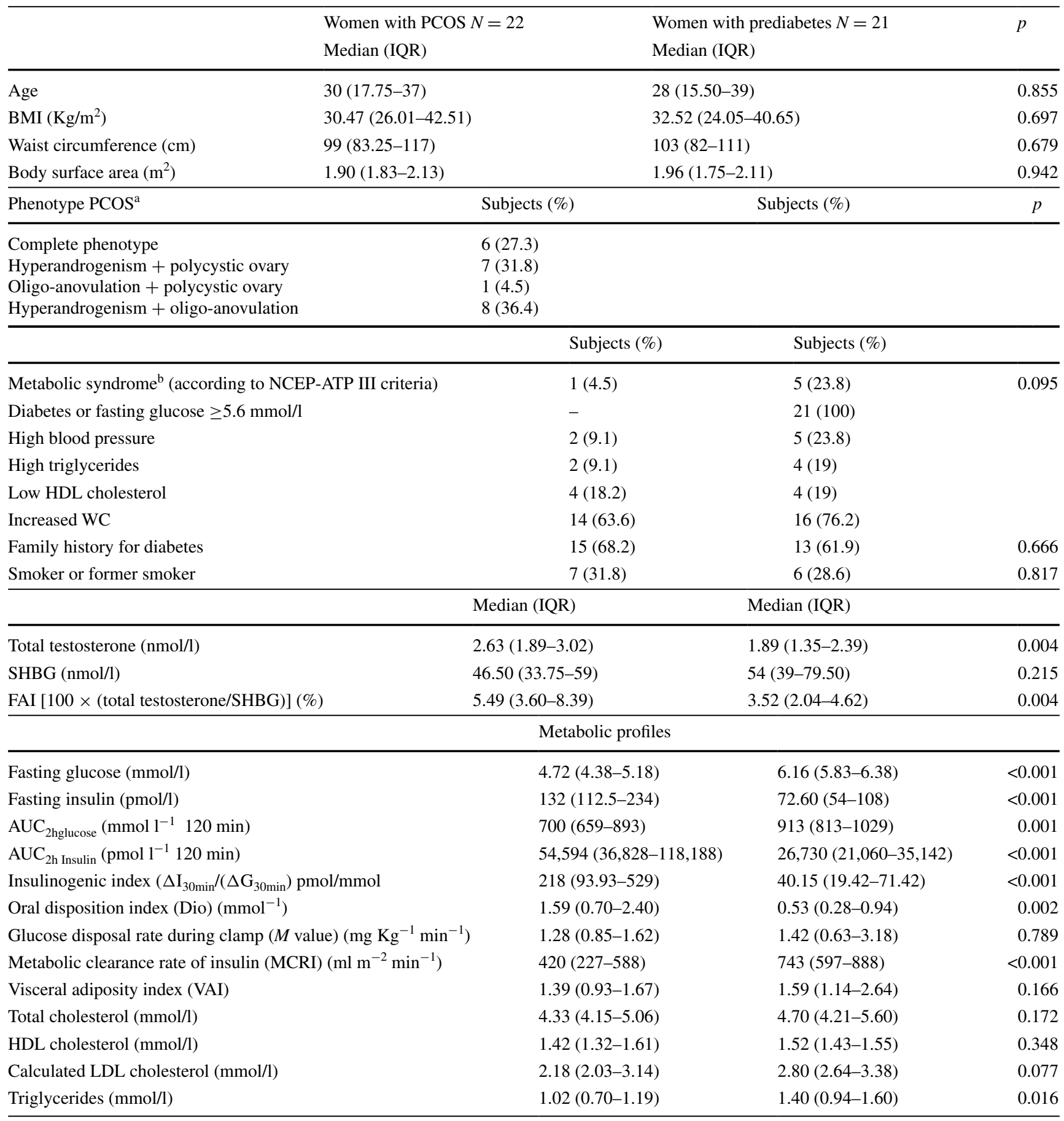

Univariate analysis: qualitative variables were analyzed through $\chi^{2}$ test or Fisher exact test; quantitative variables were analyzed through the Mann-Whitney $U$ test

${ }^{\text {a }}$ Rotterdam criteria

b According to adult treatment panel (ATP) III criteria

At a multivariate analysis, we evaluated the variables that may potentially independently influence the $\mathrm{AUC}_{2 \mathrm{hInsulin}}$ : age, BMI, WC, $\mathrm{AUC}_{2 \mathrm{hGlucose}}, M$ value and MCRI. Only an independent correlation was observed with the MCRI, more marked in women with PCOS (PCOS: $\beta:-0.878 ; p<0.001$; Prediabetes: $\beta:-0.501 ; p=0.019)$ (Table 2; Fig. 2). 
Fig. 1 Differences in metabolic clearance rate of insulin (MCRI) a $M$ value, b AUC2hinsulin, $\mathbf{c}$ and oral disposition index (DIo), $\mathbf{d}$ between women with PCOS and women with prediabetes
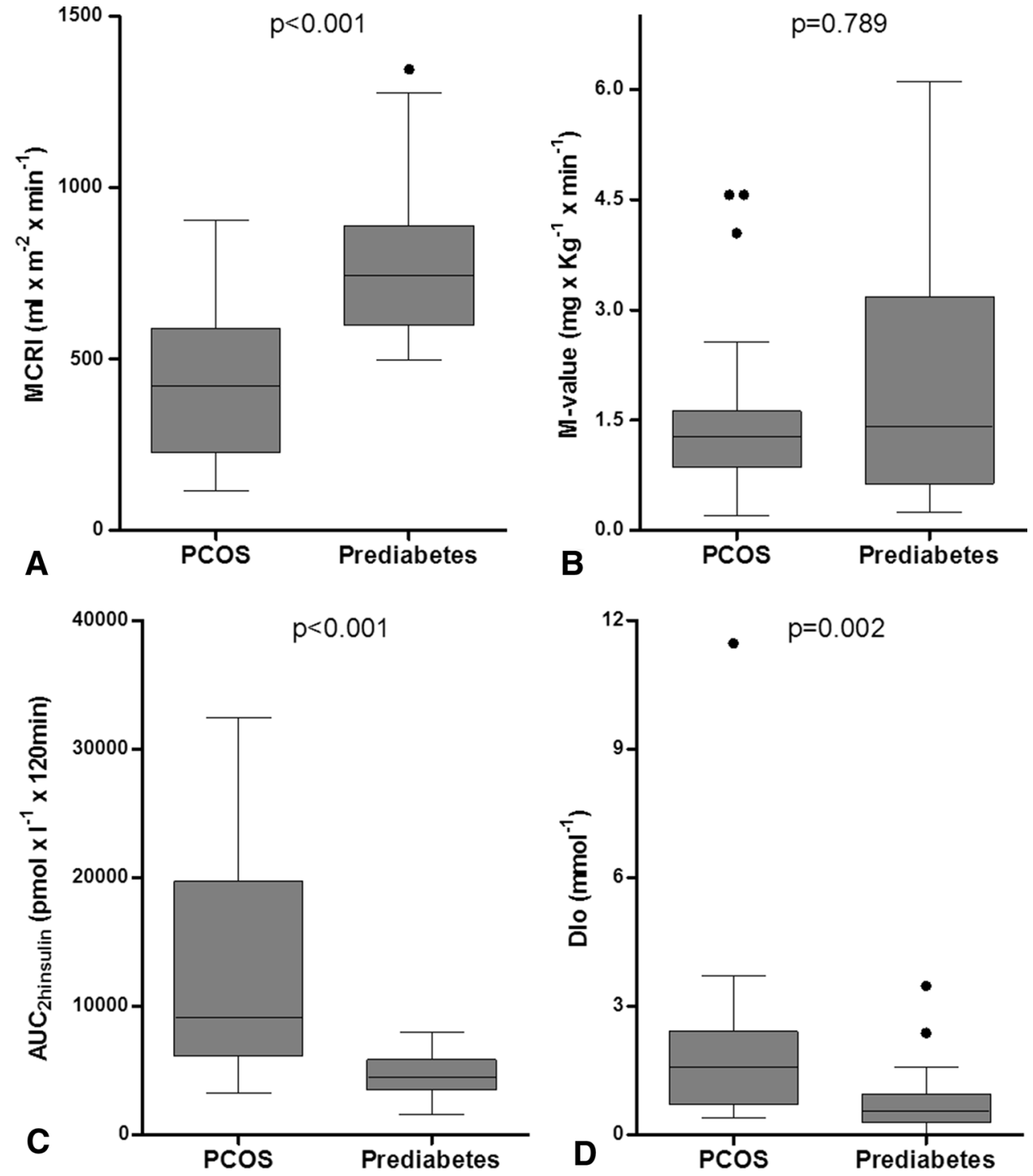

\section{Discussion}

In our study, using the gold standard for insulin resistance evaluation (Hyperinsulinemic-euglycemic clamp), we confirm that women with PCOS, despite being known to be more insulin-resistant than age-matched healthy women (regardless of BMI) [2, 31, 32], exhibit an insulin sensitivity comparable to that of the women with prediabetes and without hyperandrogenic and ovulatory disorder. This finding is also confirmed by the evidence of no difference in VAI, marker independently correlated with insulin sensitivity, between the two groups of patients. These data lead us to make some remarks on the real usefulness of the clamp in the assessment of insulin resistance of women with PCOS and on its real ability to provide information regarding all biological actions of insulin.
The clamp developed by DeFronzo et al. [26] in 1979 is a test based on the assumption that at high doses of insulin infused, the hyperinsulinemic state is sufficient to completely suppress hepatic glucose production and that there is no net change in glucose levels under steady-state conditions. Under such conditions, the rate of glucose infused is equal to the rate of whole-body glucose disposal ( $M$ value) and reflects the amount of exogenous glucose necessary to fully compensate for the hyperinsulinemia. Hence, the $M$ value reflects only the aspect of the peripheral insulininduced glucose disposal, without giving us any information about other biological actions of the hormone.

In women with PCOS the link between insulin resistance, hyperandrogenism, and ovulatory disorder is very complex: indeed, on the one hand, there is intrinsic insulin resistance characterized by insulin signaling abnormalities related to both the typical clinical features of PCOS and to common BMI-related abnormalities [33-35]. On the 
Table 2 Multivariate analysis of the hormonal, insulin-related and anthropometric parameters that independently correlate with $\mathrm{AUC}_{2 h}$ Insulin

Standardized
coefficient $\beta$$\quad$ Standard error $\quad p$

\begin{tabular}{|c|c|c|c|}
\hline \multicolumn{4}{|l|}{ Women with PCOS } \\
\hline Age & -0.014 & 95.31 & 0.904 \\
\hline BMI & 0.493 & 260.35 & 0.070 \\
\hline Waist circumference & -0.337 & 127.10 & 0.224 \\
\hline $\mathrm{AUC}_{2 \text { hglucose }}$ & -0.012 & 0.214 & 0.915 \\
\hline$M$ value & -0.100 & 767.66 & 0.387 \\
\hline MCRI & -0.878 & 4.595 & $<0.001$ \\
\hline \multicolumn{4}{|c|}{ Women with prediabetes } \\
\hline Age & 0.369 & 29.39 & 0.081 \\
\hline BMI & -0.524 & 60.32 & 0.170 \\
\hline Waist circumference & 0.698 & 31.74 & 0.080 \\
\hline AUC $_{2 \text { hglucose }}$ & 0.263 & 0.10 & 0.194 \\
\hline$M$ value & -0.299 & 194.81 & 0.155 \\
\hline MCRI & -0.501 & 1.36 & 0.019 \\
\hline
\end{tabular}

The natural logarithmic transformed values of the dependent variables included in the multiple linear regression model were used

other hand, the distinct molecular mechanisms of insulin in PCOS result in substantial hyperinsulinism causing hyperandrogenemia and anovulation $[3,4]$.

In our study, we showed that quantification of the glucose disposal rate during clamp is a technique that is not only complex, but also not very useful for understanding the pathophysiological mechanisms of the syndrome, we cannot define this evaluation useful to define the diabetes risk of a woman with PCOS, given that there are simple diagnostic alternatives derived from the OGTT that provide more specific information, such as $\mathrm{AUC}_{2 \text { hinsulin }}$ and the DIo. Indeed, women with prediabetes, while presenting a similar $M$ value compared to women with PCOS, had a significantly lower DIo. This is because DIo values well reflect the known hyperbolic relationship existing between insulin sensitivity and pancreatic insulin response [28]. Our data agree with other evidence about the limited usefulness of $M$ value evaluation and suggest that evaluation of insulin resistance alone does not fully characterize the PCOS population, since heterogeneity has been demonstrated in beta-cell activity, insulin clearance and peripheral insulin sensitivity in women with PCOS [5].

In our study, we observed that insulin levels (both fasting and after glucose stimulation), mainly in women with PCOS, were strongly influenced by the MCRI, although in women with PCOS we cannot exclude increased susceptibility to an exaggerated pancreatic response and/ or increased susceptibility in women with prediabetes to a reduced pancreatic response: hyperinsulinemia can result from decreases in insulin clearance as well as from
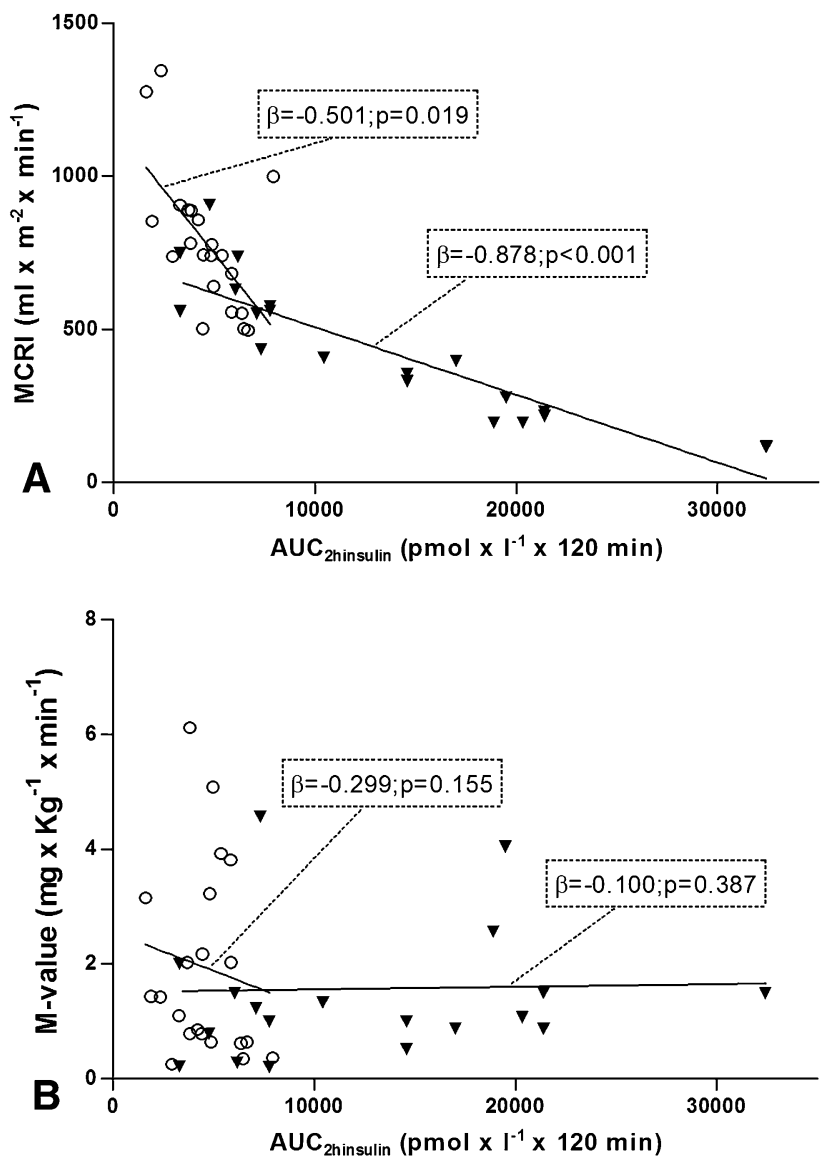

Fig. 2 Correlations between metabolic clearance rate of insulin (MCRC). a $M$ value, $\mathbf{b}$ and $\mathrm{AUC}_{2 \mathrm{hInsulin}}$ in women with PCOS (inverted triangle) and in women with prediabetes (open circle)

increases in insulin secretion [36, 37]. Also, our data partially contrast with the hypothesis that insulin clearance, being receptor-mediated, is usually decreased in insulinresistant states [36]: women with PCOS had a lower MCRI despite a comparable $M$ value to women with prediabetes.

Unfortunately, in our study we did not measure posthepatic insulin clearance; however, some evidence about normal posthepatic insulin clearance in PCOS would point to reduced hepatic clearance [38, 39].

Although there are studies in women with PCOS that have shown decreased hepatic extraction of insulin through an increase in circulating molar C-peptide/Insulin molar ratios [40, 41], we deemed it poorly useful to evaluate this molar ratio because it could be misleading; unfortunately, the assumption that the peripheral molar C-peptide/Insulin ratio can be used as a reflection of hepatic insulin extraction has not yet been experimentally validated $[42,43]$. Anyway, other studies in hyperandrogenic women also lead us to hypothesize that decreased insulin clearance in PCOS is due mainly to increased insulin hepatic extraction [44, 45]. 
Considering the fact that most of the pathophysiological studies that have addressed these issues were carried out in the 1980-90s, it would be desirable to perform future new studies on larger series for also assessing these aspects according to PCOS phenotypes.

In conclusion, our study suggests that in women with PCOS there is a degree of insulin resistance similar to that found in other insulin resistance states, such as prediabetes. What sets PCOS apart is hyperinsulinism, today still simplistically defined "compensatory"; this is mainly related to decreased insulin clearance whose specific causes and dynamics have yet to be clarified. Further prospective studies could help to identify the most reliable indicator of diabetes risk and metabolic impairment in these patients.

\section{Acknowledgments M. C. Amato-deceased.}

\section{Compliance with ethical standard}

Conflict of interest The authors declare that they have no conflict of interest.

\section{Disclosure statement The authors have nothing to disclose.}

Ethical approval All procedures performed in studies involving human participants were in accordance with the ethical standards of the institutional and/or national research committee and with the 1964 Helsinki declaration and its later amendments or comparable ethical standards.

Informed consent Informed consent was obtained from all individual participants included in the study.

\section{References}

1. Pasquali R, Gambineri A (2013) Glucose intolerance states in women with the polycystic ovary syndrome. J Endocrinol Invest 36(8):648-653

2. Stepto NK, Cassar S, Joham AE, Hutchison SK, Harrison CL, Goldstein RF, Teede HJ (2013) Women with polycystic ovary syndrome have intrinsic insulin resistance on euglycaemichyperinsulaemic clamp. Hum Reprod 28:777-784

3. Diamanti-Kandarakis E, Dunaif A (2012) Insulin resistance and the polycystic ovary syndrome revisited an update on mechanisms and implications. Endocr Rev 33:981-1030

4. Galluzzo A, Amato MC, Giordano C (2008) Insulin resistance and polycystic ovary syndrome. Nutr Metab Cardiovasc Dis 18:511-518

5. Ciampelli M, Fulghesu AM, Cucinelli F, Pavone V, Caruso A, Mancuso S, Lanzone A (1997) Heterogeneity in beta cell activity, hepatic insulin clearance and peripheral insulin sensitivity in women with polycystic ovary syndrome. Hum Reprod 12:1897-1901

6. Reaven GM (1988) Role of insulin resistance in human disease. Diabetes 37:1495-1607

7. De Fronzo RA, Ferrannini E (1991) Insulin resistance. A multifaceted syndrome responsible for NIDDM, obesity, hypertension, dyslipidemia, and atherosclerotic cardiovascular disease. Diabetes Care 14:173-194

8. Kahn SE, Prigeon RL, McCulloch DK, Boyko EJ, Bergman RN, Schwartz MW, Neifing JL, Ward WK, Beard JC, Palmer JP
(1993) Quantification of the relationship between insulin sensitivity and $\beta$-cell function in human subjects. Evidence for a hyperbolic function. Diabetes 42:1663-1672

9. Bergman RN, Phillips LS, Cobelli C (1981) Physiologic evaluation of factors controlling glucose tolerance in man: measurement of insulin sensitivity and $\beta$-cell glucose sensitivity from the response to intravenous glucose. J Clin Invest 68:1456-1467

10. Watanabe RM, Valle T, Hauser ER, Ghosh S, Eriksson J, Kohtamäki K, Ehnholm C, Tuomilehto J, Collins FS, Bergman RN, Boehnke M (1999) Familiality of quantitative metabolic traits in Finnish families with non-insulin-dependent diabetes mellitus. Finland-United States investigation of NIDDM Genetics (FUSION) Study investigators. Hum Hered 49:159-168

11. Lyssenko V, Jonsson A, Almgren P, Pulizzi N, Isomaa B, Tuomi T, Berglund G, Altshuler D, Nilsson P, Groop L (2008) Clinical risk factors, DNA variants, and the development of type 2 diabetes. N Engl J Med 359:2220-2232

12. Boesgaard TW, Grarup N, Jørgensen T, Borch-Johnsen K, Hansen T, Pedersen O (2010) Variants at DGKB/TMEM195, ADRA2A, GLIS3 and C2CD4B loci are associated with reduced glucose-stimulated $\beta$ cell function in middle-aged Danish people. Diabetologia 53:1647-1655

13. Lorenzo C, Wagenknecht LE, Rewers MJ, Karter AJ, Bergman RN, Hanley AJ, Haffner SM (2010) Disposition index, glucose effectiveness, and conversion to type 2 diabetes. Diabetes Care 33:2098-2103

14. Weyer C, Bogardus C, Mott DM, Pratley RE (1999) The natural history of insulin secretory dysfunction and insulin resistance in the pathogenesis of type 2 diabetes mellitus. J Clin Invest 104:787-794

15. Jaspan J, Polonsky K (1982) Glucose ingestion in dogs alters the hepatic extraction of insulin. In vivo evidence for a relationship between biologic action and extraction of insulin. J Clin Invest 69:516-525

16. Honey RN, Price S (1979) The determinants of insulin extraction in the isolated perfused rat liver. Horm Metab Res 11:111-117

17. Rubenstein AH, Pottenger LA, Mako M, Getz GS, Steiner DF (1972) The metabolism of proinsulin and insulin by the liver. J Clin Invest 51:912-921

18. Harding PE, Bloom G, Field JB (1975) Effect of infusion of insulin into portal vein on hepatic extraction of insulin in anesthetized dogs. Am J Physiol 228:1580-1588

19. Rotterdam ESHRE/ASRM-Sponsored PCOS consensus workshop group (2004) Revised 2003 consensus on diagnostic criteria and long-term health risks related to polycystic ovary syndrome (PCOS). Hum Reprod 19:41-47

20. Ferriman D, Gallwey JD (1961) Clinical assessment of body hair growth in women. J Clin Endocrinol Metab 21:1440-1447

21. Vermeulen A, Verdonck L, Kaufman JM (1999) A critical evaluation of simple methods for the estimation of free testosterone in serum. J Clin Endocrinol Metab 84:3666-3672

22. Amato MC, Verghi M, Galluzzo A, Giordano C (2011) The oligomenorrhoic phenotypes of polycystic ovary syndrome are characterized by a high visceral adiposity index: a likely condition of cardiometabolic risk. Hum Reprod 26:1486-1494

23. Christensen JT, Boldsen J, Westergaard JG (1997) Ovarian volume in gynecologically healthy women using no contraception, or using IUD or oral contraception. Acta Obstet Gynecol Scand 76:784-789

24. Expert Panel on Detection, Evaluation, and Treatment of High Blood Cholesterol in Adults (2001) Executive summary of the third report of the National Cholesterol Education Program (NCEP) 2001 expert panel on detection, evaluation, and treatment of high blood cholesterol in adults (adult treatment panel III). JAMA 285:2486-2497

25. American Diabetes Association (2014) Standards of medical care in diabetes-2014. Diabetes Care 37(Suppl 1):S14-S80 
26. De Fronzo RA, Tobin JD, Andres R (1979) Glucose clamp technique: a method for quantifying insulin secretion and resistance. Am J Physiol 237:E214-E223

27. Seltzer HS, Allen EW, Herron AL Jr, Brennan MT (1967) Insulin secretion in response to glycemic stimulus: relation of delayed initial release to carbohydrate intolerance in mild diabetes mellitus. J Clin Invest 46:323-335

28. Utzschneider KM, Prigeon RL, Faulenbach MV, Tong J, Carr DB, Boyko EJ, Leonetti DL, McNeely MJ, Fujimoto WY, Kahn SE (2009) Oral disposition index predicts the development of future diabetes above and beyond fasting and 2-h glucose levels. Diabetes Care 32:335-341

29. Amato MC, Giordano C (2014) Visceral adiposity index: an indicator of adipose tissue dysfunction. Int $\mathrm{J}$ Endocrinol 2014:730827

30. Ciresi A, Amato MC, Pizzolanti G, Giordano Galluzzo C (2012) Visceral adiposity index is associated with insulin sensitivity and adipocytokine levels in newly diagnosed acromegalic patients. J Clin Endocrinol Metab 97(8):2907-2915

31. Ciresi A, Amato MC, Guarnotta V, Lo Castro F, Giordano C (2013) Higher doses of cabergoline further improve metabolic parameters in patients with prolactinoma regardless of the degree of reduction in prolactin levels. Clin Endocrinol (Oxf) 79(6):845-852

32. Amato MC, Giordano C, Galia M, Criscimanna A, Vitabile S, Midiri M, Galluzzo A, AlkaMeSy Study Group (2010) Visceral adiposity index: a reliable indicator of visceral fat function associated with cardiometabolic risk. Diabetes Care 33:920-922

33. Tam CS, Xie W, Johnson WD, Cefalu WT, Redman LM, Ravussin E (2012) Defining insulin resistance from hyperinsulinemiceuglycemic clamps. Diabetes Care 35:1605-1610

34. Dunaif A, Segal KR, Futterweit W, Dobrjansky A (1989) Profound peripheral insulin resistance, independent of obesity, in polycystic ovary syndrome. Diabetes $38: 1165-1174$

35. Ovalle F, Azziz R (2002) Insulin resistance, polycystic ovary syndrome, and type 2 diabetes mellitus. Fertil Steril 77:1095-1105
36. Corbould A, Kim Y-B, Youngren JF, Pender C, Kahn BB, Lee A, Dunaif A (2005) Insulin resistance in the skeletal muscle of women with PCOS involves intrinsic and acquired defects in insulin signaling. Am J Physiol Endocrinol Metab 288:E1047-E1054

37. Corbould A, Zhao H, Mirzoeva S, Aird F, Dunaif A (2006) Enhanced mitogenic signaling in skeletal muscle of women with polycystic ovary syndrome. Diabetes 55:751-759

38. Diamanti-Kandarakis E, Papavassiliou AG (2006) Molecular mechanisms of insulin resistance in polycystic ovary syndrome. Trends Mol Med 12:324-332

39. Hücking K, Watanabe RM, Stefanovski D, Bergman RN (2008) OGTT-derived measures of insulin sensitivity are confounded by factors other than insulin sensitivity itself. Obesity 16:1931-1938

40. Flier JS, Minaker KL, Landsberg L, Young JB, Pallotta J, Rowe JW (1982) Impaired in vivo insulin clearance in patients with severe target-cell resistance to insulin. Diabetes 31:132-135

41. Marshall S (1985) Kinetics of insulin receptor internalization and recycling in adipocytes. Shunting of receptors to a degradative pathway by inhibitors of recycling. J Biol Chem 260:4136-4144

42. Dunaif A, Sorbara L, Delson R, Green G (1993) Ethnicity and polycystic ovary syndrome are associated with independent and additive decreases in insulin action in Caribbean-Hispanic women. Diabetes 42:1462-1468

43. Pasquali R, Venturoli S, Paradisi R, Capelli M, Parenti M, Melchionda N (1982) Insulin and C-peptide levels in obese patients with polycystic ovaries. Horm Metab Res 14:284-287

44. Mahabeer S, Jialal I, Norman RJ, Naidoo C, Reddi K, Joubert SM (1989) Insulin and C-peptide secretion in nonobese patients with polycystic ovarian disease. Horm Metab Res 21:502-506

45. Polonsky KS, Rubenstein AH (1984) C-peptide as a measure of the secretion and hepatic extraction of insulin. Pitfalls and limitations. Diabetes 33:486-494 